\title{
Impairments of cerebral blood flow microcirculation in rats brought on by cardiac cessation and respiratory arrest
}

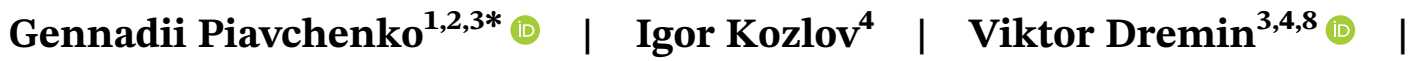 \\ Dmitry Stavtsev $^{4,6}$ | Evgeniya Seryogina ${ }^{3}$ | Ksenia Kandurova ${ }^{4}$ | \\ Valery Shupletsov $^{4}$ | Konstantin Lapin ${ }^{2}$ | Alexander Alekseyev ${ }^{3}$ | \\ Sergey Kuznetsov ${ }^{1}$ ｜ Alexander Bykov ${ }^{5}$ ｜ Andrey Dunaev ${ }^{3,4}$ ｜ Igor Meglinski ${ }^{1,2,5,7,8 *}$ (1)
}

${ }^{1}$ Department of Histology, Cytology and Embryology, I.M. Sechenov First Moscow State Medical University, Moscow, Russia

${ }^{2}$ V.A. Negovsky Scientific Research Institute of General Reanimatology, Federal Research and Clinical Centre of Intensive Care Medicine and Rehabilitology, Moscow, Russia

${ }^{3}$ Cell Physiology and Pathology Laboratory, Orel State University, Orel, Russia

${ }^{4}$ R\&D Center of Biomedical Photonics, Orel State University, Orel, Russia

${ }^{5}$ Opto-Electronics and Measurement Techniques, Faculty of Information and Electrical Engineering, University of Oulu, Oulu, Finland

${ }^{6}$ Institute of Biomedical Systems, National Research University of Electronic Technology (MIET), Zelenograd, Russia

${ }^{7}$ Immanuel Kant Baltic Federal University, Kaliningrad, Russia

${ }^{8}$ College of Engineering and Physical Sciences, Aston University,

Birmingham, UK

\section{*Correspondence}

Gennadii Piavchenko and Igor Meglinski, 8/2 Trubetskaya str., Moscow, 119991, Russia.

Email: gennadii.piavchenko@staff. sechenov.ru and i.meglinski@aston.ac.uk

\section{Funding information}

H2020 Marie Skłodowska-Curie Actions, Grant/Award Numbers: 839888, 863214; Ministry of Science and Higher Education

\begin{abstract}
The impairments of cerebral blood flow microcirculation brought on by cardiac and respiratory arrest were assessed with multi-modal diagnostic facilities, utilising laser speckle contrast imaging, fluorescence spectroscopy and diffuse reflectance spectroscopy. The results of laser speckle contrast imaging show a notable reduction of cerebral blood flow in small and medium size vessels during a few minutes of respiratory arrest, while the

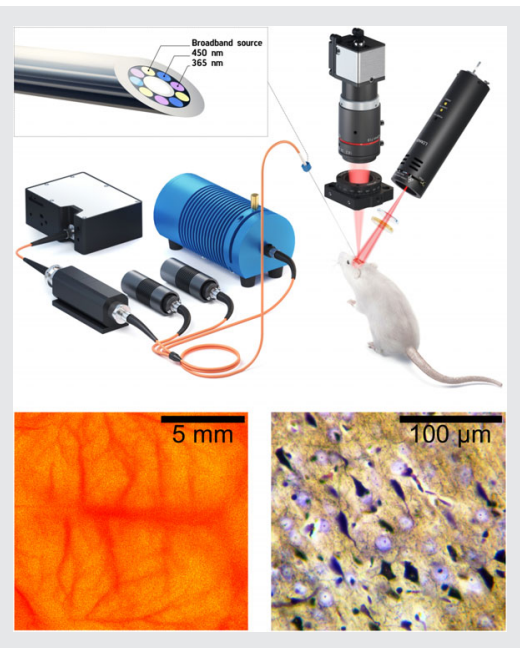
same effect was observed in large sinuses and their branches during the circulatory cessation. Concurrently, the redox ratio assessed with fluorescence spectroscopy indicates progressing hypoxia, NADH accumulation and increase of FAD consumption. The results of diffuse reflectance spectra measurements display a more rapid grow of the perfusion of deoxygenated blood in case of circulatory impairment. In addition, consequent histopathological analysis performed by using new tissue staining procedure developed in-house. It shows notably higher reduction of size of the neurons due to their wrinkling within brain tissues influenced by circulation impair. Whereas, the brain tissues altered with the respiratory arrest demonstrate focal perivascular oedema and mild hypoxic changes of neuronal morphology. Thus, the study suggests that consequences of a cessation of cerebral blood flow become more dramatic and dangerous compare to respiratory arrest.
\end{abstract}


of the Russian Federation, Grant/Award Number: 075-15-2020-926; Academy of Finland, Grant/Award Number: 326204

\section{K E Y W O R D S}

brain imaging, cardiac cessation, cerebral blood flow, laser speckle contrast, microcirculation, respiratory arrest

\section{INTRODUCTION}

Nowadays, prediction of early damage and microstructural reorganisation of cerebral tissues composition is crucial for numerous acute states, and especially those associated with a risk of brain death. The clinical death is typically caused by cessation of blood circulation and/or respiratory arrest [1]. In current study, we combined use of modern photonics-based modalities, such as laser speckle contrast imaging (LSCI), fluorescence spectroscopy (FS) and diffuse reflectance spectroscopy (DRS), the impairments of cerebral blood flow microcirculation brought by cardiac cessation and respiratory arrest are comparatively investigated.

LSCI is well-known imaging modality [2], and used extensively in a number of blood microcirculation studies, including those of tumour angiogenesis [3], cutaneous vessels reaction to allergens $[4,5]$, skin complication of diabetes [6], respiratory-related blood flow oscillations [7], and visualisation of cerebral vascular network [8]. It has been also demonstrated that with a combined application of LSCI and intravital fluorescence imaging the transcranial visualisation of brain's vascular bed can be performed in terms of blood flow within single arteries and veins [9-11].

DRS is a promising inexpensive noninvasive method that is able to show real-time dynamics of acute cerebral pathologies and to assess cerebral haemodynamics [12]. When FS and DRS are successfully utilised for monitoring metabolic and morphological changes in biological tissues in vivo [13].

In addition to the optical methods, histopathology is widely used for quantitative assessment of brain damage and direct visualisation of structural malformations associated with diseases and brain death. Histopathological analysis, used for the pathological changes within brain tissues, includes both routine staining with hemotoxylin and eosin (HE) as well as own new method of staining. In case of classical approach of HE staining, we are able to see only the most expressed pathology, making conclusion questionable. With new method we detect strong neuronal ischemia and vascular disorders as well as two types of neurons stained with cresyl violet and silver impregnation. The neuronal cells vary in structural and tinctorial properties and indicate various types of pathologies.
Thus, LSCI and DRS are used for evaluation of impairments of cerebral blood flow and blood microcirculation brought on by acute hypoxia provoked by respiration arrest and cardiac cessation. The associated variations in metabolic activities in cerebral cortex are assessed quantitatively by FS. In addition, the histopathological analysis is used to detect a hypoxic impact on the cerebral cortex in terms of structural morphological changes in postmortem brain tissues.

\section{2 | MATERIALS AND METHODS}

The experimental system combining LSCI, DRS and FS imaging/diagnostic modalities is presented in Figure 1.

The laser source $(10 \mathrm{~mW}, 785 \mathrm{~nm}$ operating wavelength, Thorlabs, Inc., USA) illuminates the area of interest through a diffuser. The back-scattered light is collected via the lens (MVL25TM23, Thorlabs, Inc., USA), and the laser speckles analysis is performed by high-resolution CMOS camera (DCC3260M, $1936 \times 1216$ pixels and 5.86-pixel size, Thorlabs, Inc., USA). To eliminate single scattering, two crossed polarizers are placed in front of camera objective and laser module (see Figure 1). The speckle contrast is counted for $40 \mathrm{~Hz}$ frame rate and $20 \mathrm{~ms}$ expose time, as [14]:

$$
C=\frac{\sigma}{\langle I\rangle_{k}},
$$

where $\sigma$ is the standard deviation for the intensity of the back scattered light, $\langle I\rangle_{k}$ is the mean intensity value, $\langle\ldots\rangle$ denotes averaging by $k$ values of the sequential stacks of images $(k=40)$. The spatial averaging of raw speckle image obtained with CMOS camera is performed utilising $5 \times 5$ pixels sliding window [10]. This approach aims to improve the signal-to-noise ratio and image quality. Speckle perfusion index (SFI) is defined as [15]:

$$
S F I=\frac{1}{2 T C^{2}},
$$

where $T$ is the exposure time of camera.

Every frame of stack is normalised by frame with the lowest mean of SFI. Thus, relative SFI is calculated as: 

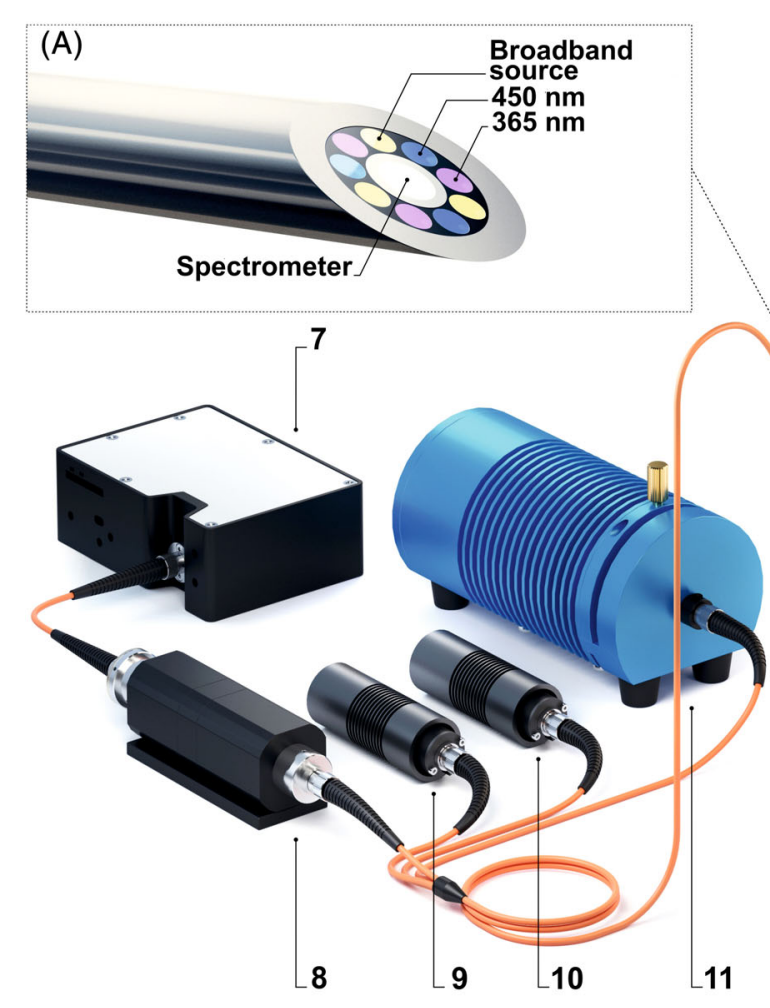

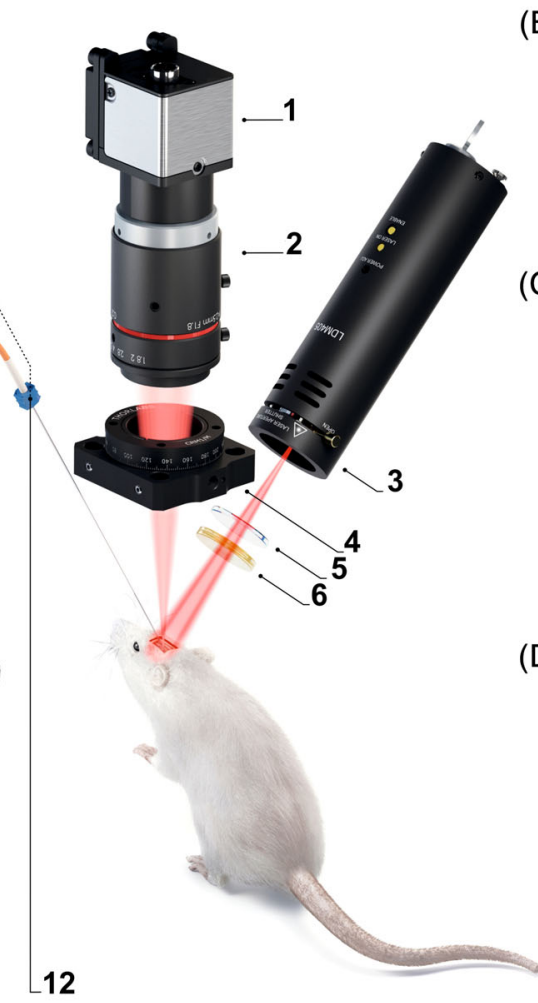

(B)

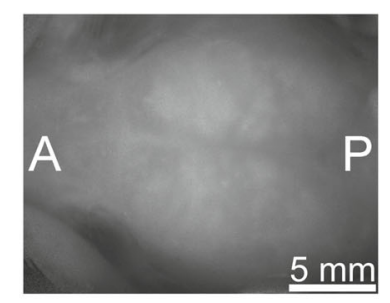

(C)
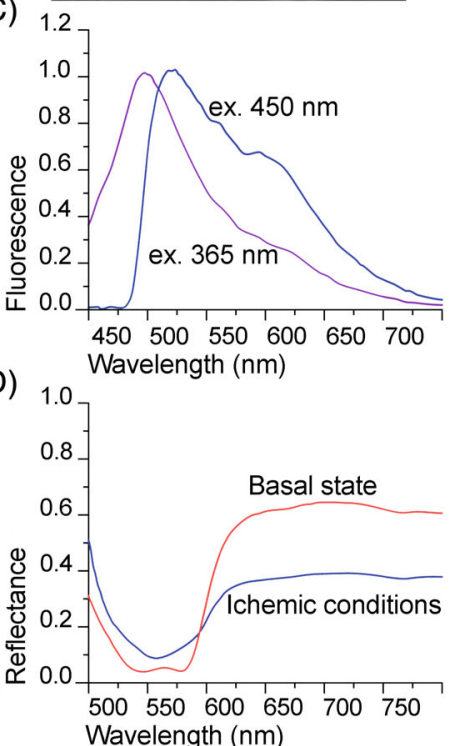

F I G U R E 1 Experimental multi-modal imaging system developed in-house (A): 1—CMOS camera, 2—camera lens, 3-785 nm laser, 4, 5-polarisers, 6-diffuser, 7-spectrometer, 8-filter holder, 9-365 nm LED, 10-450 nm laser diode, 11-halogen lamp, 12-needle probe; close-up look of the needle probe. Example of raw speckle image from rat brain surface (B), "A" denotes "anterior" and "P" denotes "posterior" anatomical directions; registered fluorescence (C) and diffuse reflectance spectra (D)

$$
\text { relative } S F I=100 \times \frac{S F I(t)}{\text { baseline } S F I} \text {, }
$$

where baseline SFI is the averaged map by first 30 frames.

LSCI measurements are recorded at the same time with FS and DRS measurements [13]. The FS mode includes $365 \mathrm{~nm}$ LED and $450 \mathrm{~nm}$ laser diode to excite the fluorescence of NADH and FAD coenzymes [16, 17]. Optical filters are used with cut off wavelengths of 400 and $490 \mathrm{~nm}$ (Thorlabs Inc., USA), respectively, to attenuate the backscattered light. DRS mode includes 360 to $2400 \mathrm{~nm}$ tungsten halogen light source HL-2000-FHSA (Ocean Insight, USA). The FS and DRS spectra are recorded by "FLAME" spectrometer (Ocean Insight, USA) and analysed in the range of 400 to $900 \mathrm{~nm}$. The custom application is developed in MATLAB (MathWorks, Inc., USA) environment for data recording, saving and controlling the setup.

The computational analysis of spatial localisation of the LSCI and FS detected signals [18, 19] at $785 \mathrm{~nm}$ shows that the effective penetration depth in rodents tissues lies in a range $\sim 0.5-0.7 \mathrm{~mm}$ ). Since the thickness of skin and skull in rodents is about $\sim 0.5 \mathrm{~mm}$, the young rats have been selected for noninvasive transcranial cerebral imaging.

To deliver the optical radiation into the cortex, a custom fibre-optic probe is applied (see Figure 1). The probe has $1 \mathrm{~mm}$ diameter and $20^{\circ}$ bevel to ensure a good contact as well as not to damage brain tissues significantly. The probe contains 10 optical fibres (0.22 NA). The central one ( $200 \mu \mathrm{m}$ in diameter) the light from the tissue surface to the spectrometer [17], whereas the surrounding fibres $(100 \mu \mathrm{m}$ in diameter, three fibres per light source). To avoid uncertainties in spectral measurements associated with the local suppression of superficial soft tissues $[20,21]$ the probe is placed close to the skin surface without touching it. The FS and DRS spectra are recorded during 1.5 and $0.5 \mathrm{~s}$, respectively. To avoid a photobleaching effect, fluorescence excitation sources have been turned off between the measurements. The examples of registered raw speckle image, FS and DRS are shown in Figure 1B-D. Both 2 weeks old and 2 months old male Wistar rats were used in the experiments. All animals were kept in controlled environmental conditions (20-26 $\mathrm{C}$ of temperature, $50-60 \%$ of humidity, $12 \mathrm{~h}$ day-night cycle and 10 times air exchange 


\section{Respiratoty arrest model}

Propofol $0.3 \mathrm{ml}$<smiles>CC(C)c1cccc(C(C)C)c1O</smiles>

Non-depolarizing muscle relaxant (cisatracurium) in a lethal dose

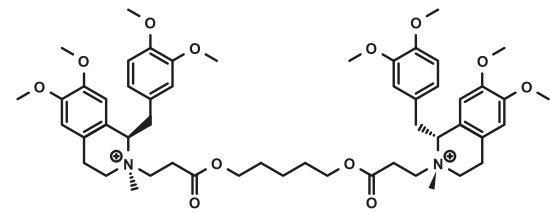

Immediately causes a spasm of the respiratory muscles and respiratory arrest
Cardiac cessation model

Lidocaine solution (2\%) in a dose of $1 \mathrm{ml}$<smiles>CCN(CC)CC(=O)Nc1c(C)cccc1C</smiles>

Immediately causes a cardiac arrest

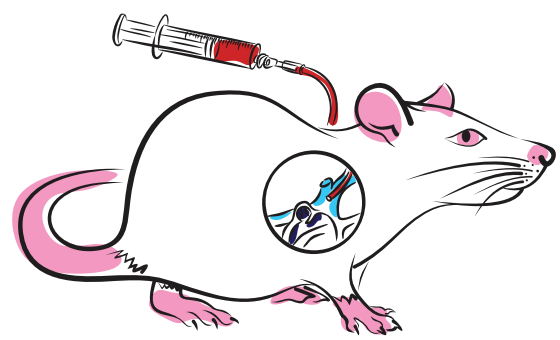

FI G URE 2 Schematic presentation of respiratory and cardiac cessation. After placement in the stereotaxic apparatus the animal was administrated with drugs that caused immediate respiratory arrest (propofol followed by cisatracurium) or acute cardiac cessation (lidocaine solution)

\section{0:00}
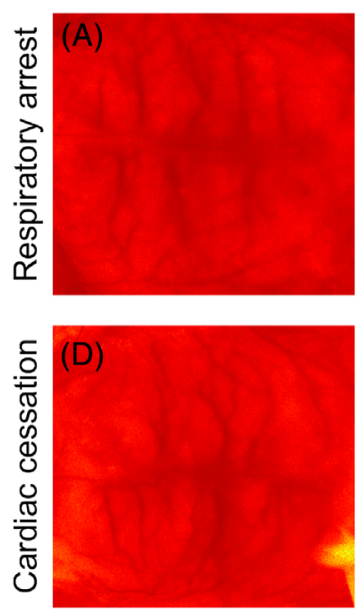

01:00

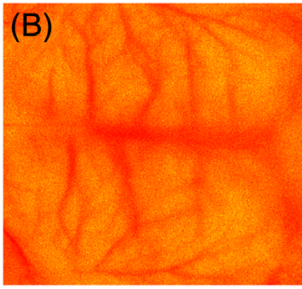

(E)

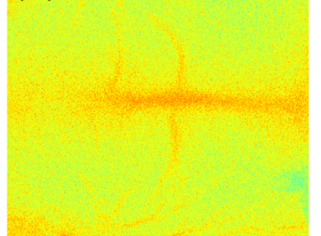

02:00

(C)

F)
.40

0.35

0.30

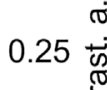

0.20

$0.15 \frac{7}{8}$

0.10 के

0.05
F I G URE 3 Blood flow dynamics in time. The upper row corresponds to respiration impair $(\mathrm{A}, \mathrm{B}, \mathrm{C})$, and the lower one to cardiac cessation (D, E, F). Notable changes appear from 1st minute of the recording $(\mathrm{B}, \mathrm{E})$. Video for blood flow dynamics is available (Rat brain. Cardiac cessation and respiratory arrest, 191.4 Mb, .avi [URL: https://doi.org/10. 5281/zenodo.4739049]) per hour) in accordance with the principles of Good Laboratory Practice [22].

The animals were anaesthetized by an intramuscular injection of Zoletil drug (dose-25 mg/kg, Vibrac, France) and catheterized into right external jugular vein with PM-60 polymer catheter (SciCat, Russia, $d_{\text {ext }}=0.8 \mathrm{~mm}, d_{\text {int }}=0.4 \mathrm{~mm}$ ). The jugular veins catheterization approach is anatomically easily accessible with a minimal influence on the circulatory system, and is also accompanied by a lower risk of blood loss. The injection of drug into the jugular vein in comparison with the tail vein provides better compatibility [23]. After the anaesthesia, the animal is placed on a heating table to control body temperature and is fixed in a 3D-printed animal body holder, developed in-house, to avoid movement artefacts [24]. All the manipulations with the animals are approved by the ethical committee of the Orel State University named after I.S. Turgenev (protocol No. 10, 16 October 2018).

The experimental models of respiratory and cardiac arrest are performed for the corresponding animals groups by the injection of drugs combination via catheter (Figure 2). We have checked the heart rate (to confirm the cardiac cessation) with an electrode detecting the heart beats during the experiment. We also counted the respiratory rate (number of diaphragm contractions) by a visual registration of breathing acts. As far as cisatracurium immediately causes a respiratory muscles spasm, any last diaphragm contraction has been considered as a breathing stop in the animal.

After LSCI, FS, DRS imaging and spectra recording, brains of all the animals were fixed in buffered formalin. Paraffin embedded sections of $5 \mu \mathrm{m}$ thickness are prepared 
FI G URE 4 The LSCI images observed during respiratory arrest (A) and cardiac cessation (B) arrest with the selected areas of particular groups of vessels: 1 -veins of medium calibre, $2-$ vessels of microcirculation, 3-venous sinus. Corresponded temporal variations of relative SFI (C, D, E) observed during respiratory (blue) and cardiac (red) arrest in the selected areas: 1, 2,

3. Anatomical direction signs are same as Figure 1B

FIGURE 5 The results of FS and DRS measurements: (A) redox ratios of fluorescence intensity (maxima associated with NADH and FAD); (B) DRS ratio obtained at 540 and $805 \mathrm{~nm}$ during respiration arrest (C) and cardiac cessation (D)
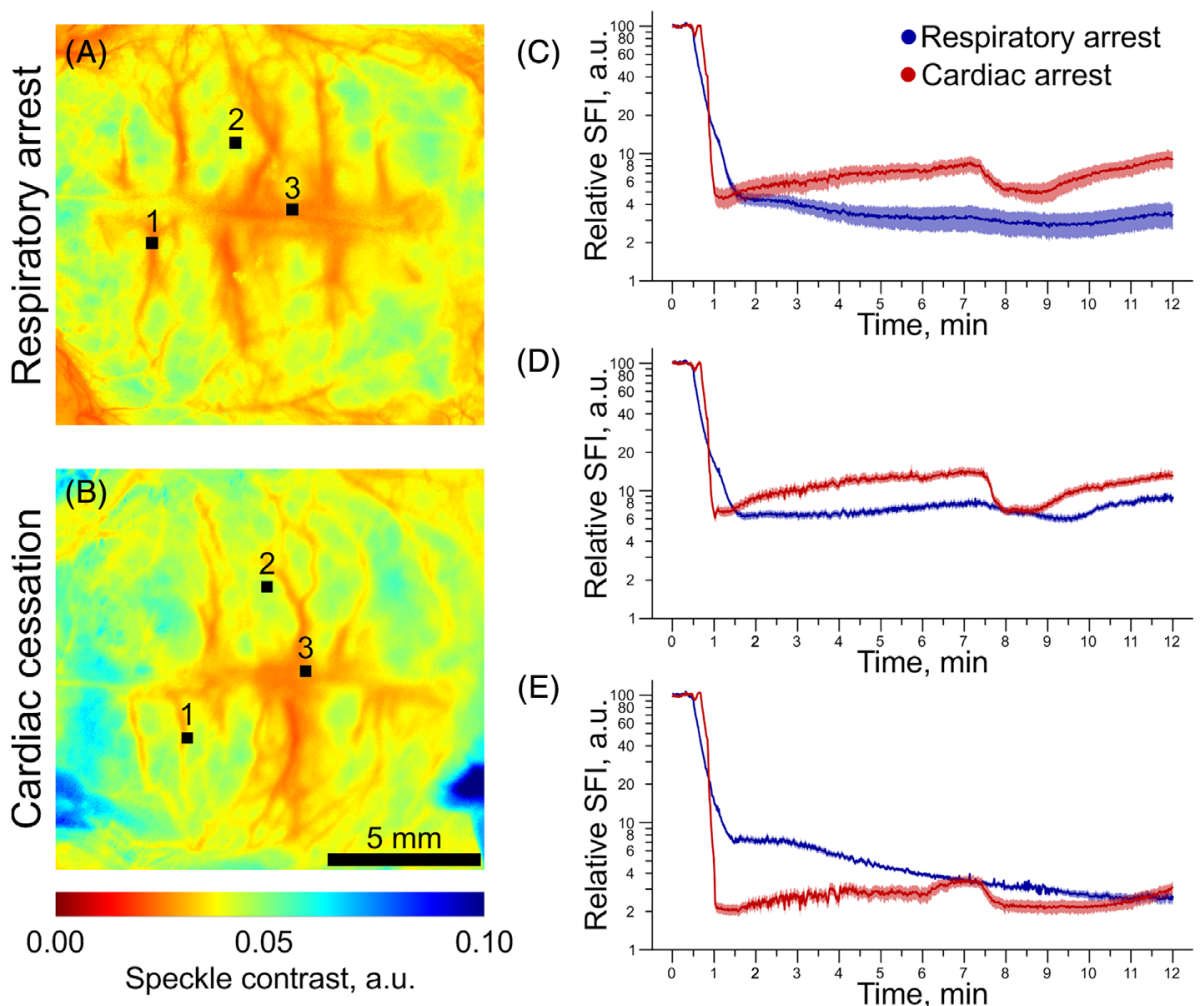

(E)
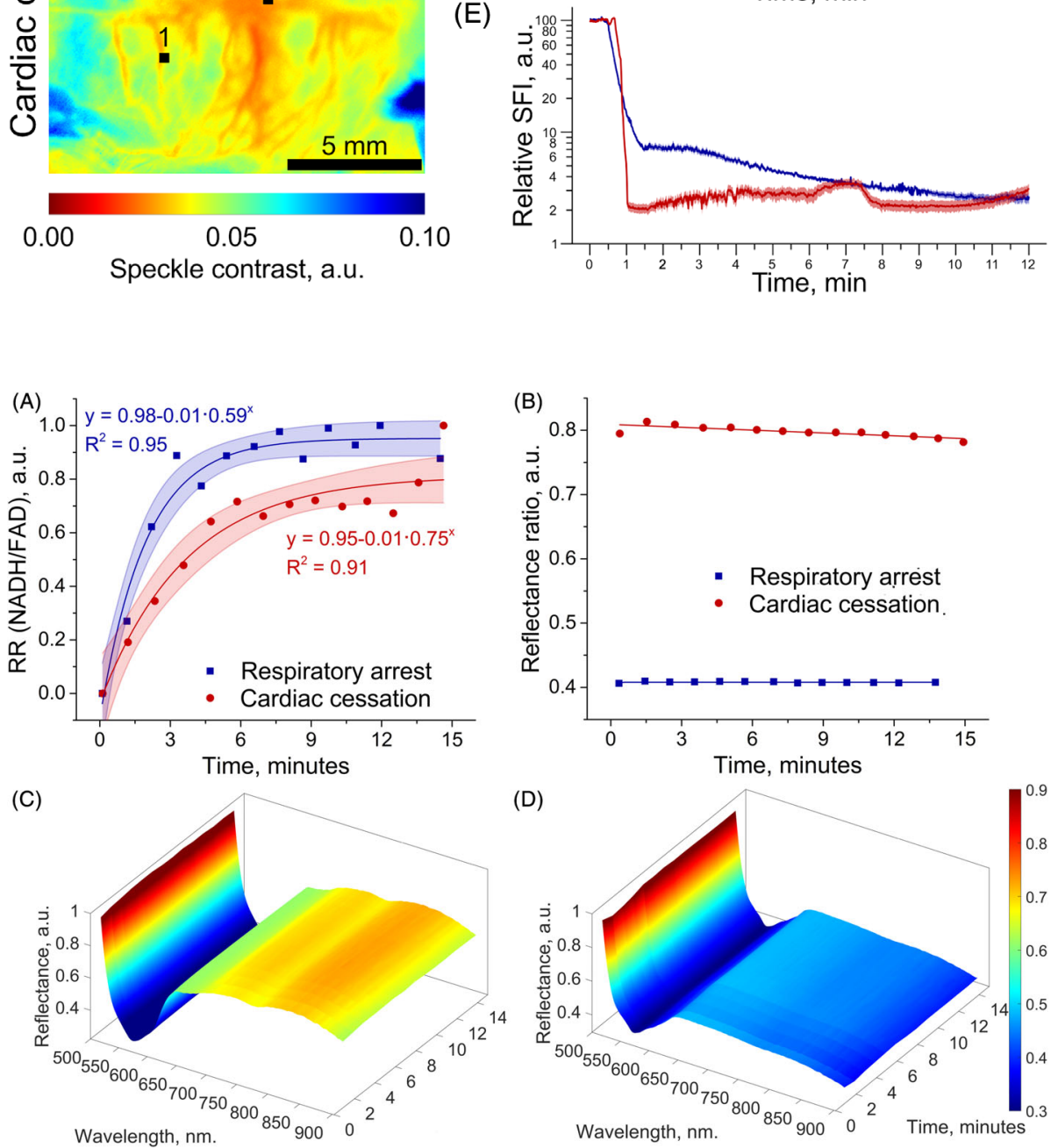

with Microm HM 450 microtome (Microm International $\mathrm{GmbH}$, Germany) and stained with HE by standard protocols [25] and with our own patented Nissl staining and silver impregnation combined method [26]. This new approach allows to see both silver impregnated and cresyl violet stained neurons with different structural and functional properties. The prepared slides were studied with
Leica DM4 M Microscope (Leica Microsystems GmbH, Germany) focusing on finding the possible ischemia and vascular pathology changes in the motor cortex area, as this region mostly contributes in the motor behaviour regulation (functioning as motor-related areas of cortex [27]). Paxinos and Watson Rat Brain Stereotaxic Atlas [28] is used as a reference guideline. 

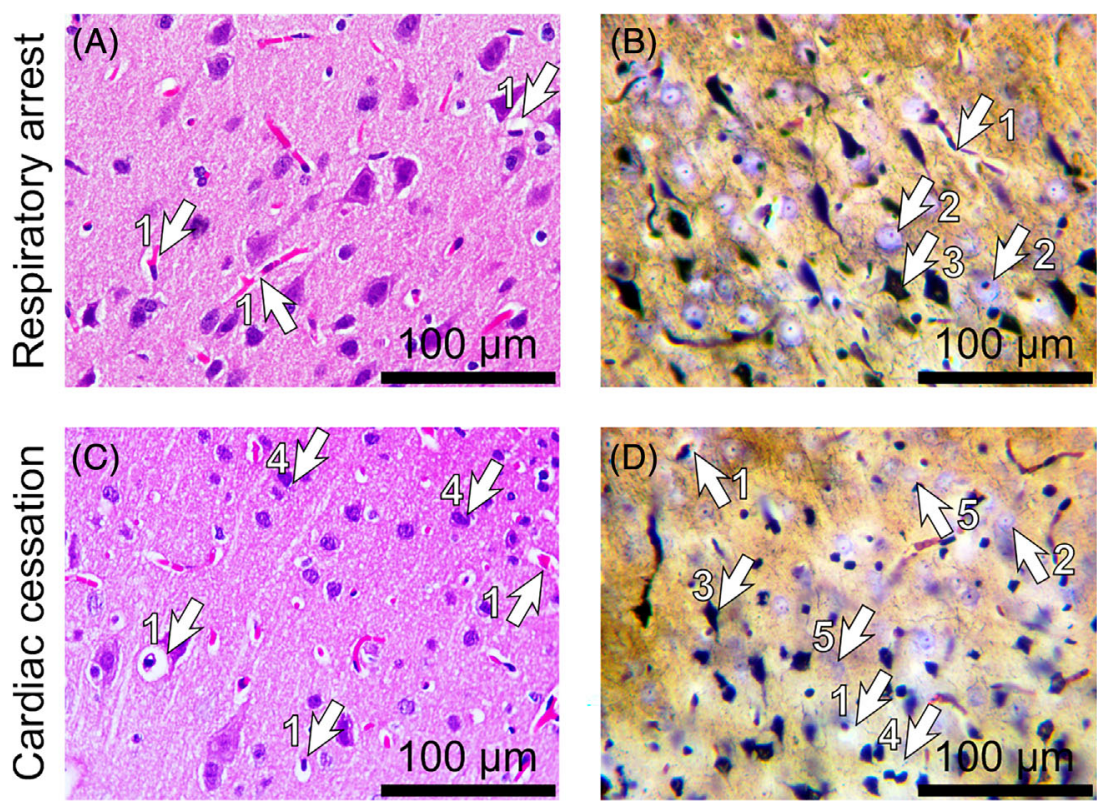

F I G URE 6 The results of histopathological analysis of coronal brain tissue sections in vitro: $(\mathrm{A}, \mathrm{C})$ rat motor cortex stained with haematoxylin and eosin; (B, D) rat motor cortex stained with method developed in-house utilising cresyl violet and silver impregnation combination. The circles highlight vessels with perivascular oedema (1), cresyl violet stained neurons with nucleoli (2), totally impregnated neurons (3), necrobiotic cells (4), ghost-like cells (5)

\section{3 | RESULTS AND DISCUSSION}

The impairments of cerebral blood flow microcirculation in cardiac cessation and respiratory arrest are presented in Figure 3. Anatomical direction signs are same as Figure 1B. The cerebral blood flow microcirculation measured with LSCI at normal conditions are seen in Figure 3A,D. Figure 3B,C, E show the map of cerebral blood flow microcirculation after $1 \mathrm{~min}$ of respiratory arrest and cardiac cessation, respectively, whereas Figure 3C,F represent same mappings after 2 min passed.

Temporal variations of relative SFI observed for the areas which correspond to the large (venous sinus, $\sim 100 \mu \mathrm{m}$ in diameter), medium (veins of medium calibre, $\sim 30-40 \mu \mathrm{m}$ in diameter) and small (vessels of microcirculation, $\sim 2-10 \mu \mathrm{m}$ in diameter) cerebral blood vessels (Figure 4A,B) are presented in Figure 4C-E. As one can see, the relative SFI becomes steady for the first $30 \mathrm{~s}$ of measurements for both models. While, for the respiration arrest it takes $1 \mathrm{~min}(0.5-1.5 \mathrm{~min})$ to get an extensive decrease of the relative SFI while in case of cardiac cessation it occurs in few seconds (see Figure 4). Further, a monotonic decrease of the relative SFI is observed with a flop between 6 and $8 \mathrm{~min}$ of observation. The obtained results suggest that for the respiration arrest the blood flow becomes most influenced first in medium and large size vessels (see Figure 4C and E, respectively), with the following monotonic decrease of flow. Whereas in small vessels the intensity of blood microcirculation decreasing after first minute is preserved both in case of respiratory arrest and cardiac cessation (see Figure 4D). The blood flow in the venous sinus is dropped significantly and much faster in case of cardiac cessation (see Figure 4E).
The results of FS and DRS measurements analysed with Origin Pro (OriginLab Corp., USA) and MATLAB, are presented in Figure 5. In the FS measurements, the maxima of emission intensity at 490 to $510 \mathrm{~nm}$ (for the excitation wavelength of $365 \mathrm{~nm}$ ) and 510 to $530 \mathrm{~nm}$ (for $450 \mathrm{~nm}$ ), respectively, are caused by the dominant contribution of NADH $\left(\mathrm{IF}_{\mathrm{NADH}}\right)$ and FAD $\left(\mathrm{IF}_{\mathrm{FAD}}\right)$. The redox ratio (RR) [29] defined as [30, 31]:

$$
R R=\frac{I F_{N A D H}}{I F_{F A D}}
$$

is used to evaluate a metabolic changes of brain tissues associated with the impairments of cerebral blood flow microcirculation brought by cardiac cessation and respiratory arrest.

The RR demonstrates a progressive increase in hypoxia, an accumulation of NADH and increased FAD consumption. Trends identification for the full duration of the experiment is carried out using an exponential fitting with a R-square evaluation. These results are well agreed with the results of an alternative study [32, 33]. The changes of RR are more expressed for the case of respiration arrest, and showing less influence in the acute cerebral cortex ischemia due to cardiac cessation. The acute intensity changes are observed for the first $5 \mathrm{~min}$ after the heart arrest. The increase of RR is caused by growth of NADH-associated fluorescence and decrease of FAD. The obtained result suggests that blood circulation fail leads to a more acute progression of ischemia and to following metabolic derangements in brain cortex.

The DRS measurements at the range of wavelengths associated with oxy- $(540 \mathrm{~nm})$ and deoxy- $(560 \mathrm{~nm})$ 
absorption peaks of haemoglobin, as well as at the isobestic point of $805 \mathrm{~nm}$ of oxy- and doxy-absorption provides an extra opportunity to assess the changes in blood content [34]. The results of DRS measurements show relatively steady dynamics of the reflectance ratio between maximum and minimum values, that do not exceed 5\% (see Figure 5B). Both cases demonstrate reflectance changes (see Figure 5C,D) that are associated with the decrements of oxyhemoglobin within the sampling volume. These changes have no significant impact on the results of FS which are influenced by metabolic changes due to hypoxia $[35,36]$.

In addition to the results of LSCI, FS and DRS measurements mentioned above, the histopathology analysis of brain tissues is presented in Figure 6. In the brain cortex sections of the first respiration arrest group, a focal perivascular oedema and mild hypoxic changes of neuronal morphology are clearly seen (see Figure 6A). In the case of cardiac cessation, the brain cortex contains a significant number of hyperchromic wrinkled neurons, glial nodules at the site of necrotic neurons, as well as pericellular oedema in all tissue sections (see Figure 6C).

For specimens stained with the new approach developed in-house, in both cases the pathological changes in nervous tissue morphology are observed (see Figure 6B,D). The results of histopathology analysis for the group of animals with cardiac cessation also show a more considerable size reduction of impregnated and cresyl violet stained neurons due to their wrinkling. There are also initial stages of anucleated pale "ghost-like" neurons forming, a less distinct neuropil structure, a decreased basophilia of cellular cytoplasm (due to the reduction of activity in synthetic apparatus, or chromatophilic substance), a less smoothed outline of the neurons as well as dark impregnated neurons, (see Figure 6B,D). The new staining approach allows to compare the changes in neuronal morphology provoked by impairments of cerebral blood flow microcirculation brought by cardiac cessation and respiratory arrest.

\section{4 | SUMMARY AND CONCLUSIONS}

In acute respiratory arrest there is a gradual increase of hypoxia as well as a slowdown of haemodynamics. For acute circulatory impair we observe an immediate cease of oxygenated blood supply to the brain cortex. We conclude that acute cardiovascular impair (e.g., sudden cardiac arrest) is more life-threatening and leads to a faster increase of neuronal metabolism deficiency in comparison with acute respiratory impair. After long time hypoxia, the biochemical processes shift to the low energy mode and provide a resistance to hypoxia until a state of full anoxia is achieved. The obtained results are well agreed with known literature data. Changes of FS intensities, which lead to a RR increase, might be noticed after $5 \mathrm{~min}$, while the trend for DRS ratios remains almost the same, especially in the case of respiration impair. Blood circulation in vessels of cerebral cortex stops in case of respiratory arrest after about 2 min while in heart arrest it happens after about $1 \mathrm{~min}$. Changes in the brain tissue oxygen saturation for heart arrest become evident during the first $5 \mathrm{~min}$, but for acute breathing fail there are almost no changes. Heart arrest manifests by a more acute hypoxic state in cerebral cortex comparing to what is happening when breathing is impaired. So, acute cardiovascular impair is more life-threatening. It causes a rapid increase in neuronal metabolic deficiency comparing to acute respiratory impair. This may serve to personalise the management of patients after acute circulatory and respiratory arrest for prognosis and rehabilitation. The utilised multimodal photonics-based approach of brain tissue functional monitoring may allow to bring a complex assessment with laser speckle and spectroscopy methods for evaluation of brain metabolism changes in neurosurgical patients.

\section{ACKNOWLEDGMENTS}

The authors disclose receipt of financial support for the research, authorship, and/or publication of this article as following: Gennadii Piavchenko and Sergey Kuznetsov acknowledge the support from the Ministry of Science and Higher Education of the Russian Federation within the framework of State support for the creation and development of World-Class research Centres "Digital Biodesign and Personalized Healthcare" No 075-15-2020926. Viktor Dremin kindly acknowledges personal support from the European Union's Horizon 2020 research and innovation program under the Marie SkłodowskaCurie grant agreement No. 839888. Andrey Dunaev and Igor Meglinski acknowledge funding from the Academy of Finland (grant No. 326204). This study has been also partially supported by the European Union's Horizon 2020 research and innovation programme under grant agreement No. 863214-NEUROPA project.

\section{CONFLICT OF INTEREST}

The authors declare no conflict of interest.

\section{DATA AVAILABILITY STATEMENT}

Data sharing is not applicable to this article as no new data were created or analyzed in this study.

\section{ORCID}

Gennadii Piavchenko (1) https://orcid.org/0000-0001-77823468 
Viktor Dremin (10) https://orcid.org/0000-0001-6974-3505

Igor Meglinski (1D https://orcid.org/0000-0002-7613-8191

\section{REFERENCES}

[1] A. Walker, Cerebral death, 3rd ed., Urban \& Schwarzenberg, Baltimore, MD 1985.

[2] W. Heeman, W. Steenbergen, G. van Dam, E. C. Boerma, J Biomed Opt. 2019, 24(8), 080901.

[3] V. Kalchenko, N. Madar-Balakirski, I. Meglinski, A. Harmelin, J. Biophotonics 2011, 4(9), 645.

[4] V. Kalchenko, Y. Kuznetsov, D. Preise, I. Meglinski, A. Harmelin, J Biomed Opt. 2014, 19(6), 060502.

[5] V. Kalchenko, I. Meglinski, A. Sdobnov, Y. Kuznetsov, A. Harmelin, J Biomed Opt 2019, 24(6), 060501.

[6] E. Zharkikh, V. Dremin, E. Zherebtsov, A. Dunaev, I. Meglinski, J. Biophoton 2020, 13(10), e202000203.

[7] I. Mizeva, E. Zharkikh, V. Dremin, E. Zherebtsov, I. Makovik, E. Potapova, A. Dunaev, Microvasc. Res. 2018, 120, 13.

[8] V. Kalchenko, D. Israeli, Y. Kuznetsov, A. Harmelin, Sci. Rep. 2014, 4(1), 5839.

[9] V. Kalchenko, D. Israeli, Y. Kuznetsov, I. Meglinski, A. Harmelin, J. Biophotonics 2015, 8(11-12), 897.

[10] V. Kalchenko, A. Sdobnov, I. Meglinski, Y. Kuznetsov, G. Molodij, A. Harmelin, Photonics 2019, 6(3), 80.

[11] G. Molodij, A. Sdobnov, Y. Kuznetsov, A. Harmelin, I. Meglinski, V. Kalchenko, Phys. Med. Biol 2020, 65(7), 075007.

[12] R. M. Forti, M. Katsurayama, J. Menko, L. Valler, A. Quiroga, A. L. E. Falcão, L. M. Li, R. C. Mesquita, Front. Med. 2020, 7, 147.

[13] V. Dremin, E. Potapova, E. Zherebtsov, K. Kandaurova, V. Shupletsov, A. Alekseyev, A. Mamoshin, A. Dunaev, Sci. Rep. 2020, 10, 14200.

[14] J. D. Briers, S. Webster, J Biomed Opt. 1996, 1(2), 174.

[15] C. Crouzet, R. H. Wilson, A. Bazrafkan, M. H. Farahabadi, D. Lee, J. Alcocer, B. J. Tromberg, B. Choi, Y. Akbari, Biomed. Opt. Express 2016, 7(11), 4660.

[16] A. C. Croce, G. Bottiroli, Eur J Histochem 2014, 58(4), 2461.

[17] A. V. Dunaev, V. V. Dremin, E. A. Zherebtsov, I. E. Rafailov, K. S. Litvinova, S. G. Palmer, N. A. Stewart, S. G. Sokolovski, E. U. Rafailov, Med. Eng. Phys. 2015, 37(6), 574.

[18] Y. L. Kuznetsov, V. V. Kalchenko, N. G. Astaf'eva, I. V. Meglinski, Quant. Electron 2014, 44(8), 713.

[19] V. Dremin, E. Zherebtsov, A. Bykov, A. Popov, A. Doronin, I. Meglinski, Appl. Optics 2019, 58(34), 9398.

[20] A. Popov, A. Bykov, I. Meglinski, J Biomed Opt 2017, 22(11), 110504.

[21] I. Mizeeva, E. Potapova, V. Dremin, E. Zherebtsov, M. Mezentsev, V. Shuleptsov, A. Dunaev, Clin. Hemorheol. Microcirc. 2019, 72(3), 259.
[22] OECD Series on principles of good laboratory practice and compliance monitoring. https://www.oecd.org/officialdocuments/ publicdisplaydocumentpdf $/$ ?cote $=$ env $/ \mathrm{mc} / \mathrm{chem} \% 2898 \% 2917 \&$ doclanguage $=$ en.

[23] K. V. Thrivikraman, R. L. Huot, P. M. Plotsky, Brain Res. Brain Res. Protoc 2002, 10(2), 84.

[24] I. M. Gryadunov, G. A. Piavchenko, I. O. Kozlov, E. S. Seryogina, S. L. Kuznetsov, RU patent 200025U1 (2020).

[25] G. A. P'yavchenko, L. I. Shmarkova, V. I. Nozdrin, Neurosci. Behav. Physiol 2016, 46(3), 270.

[26] G. A. Piavchenko, D. Pranab, N. S. Novikova, V. I. Nozdrin, RU patent 2666256C2 (2018).

[27] X. Li, M. A. Krol, S. Jahani, D. A. Boas, H. Tager-Flusberg, M. A. Yücel, Sci. Rep 2020, 10(1), 10965.

[28] G. Paxinos, C. Watson, The Rat Brain in Stereotaxic Coordinates: Hard Cover Edition, Elsevier, San Diego, CA 2006.

[29] B. Chance, B. Schoener, R. Oshino, F. Itshak, Y. Nakase, J. Biol. Chem 1979, 254(11), 4764.

[30] J. H. Ostrander, C. M. McMahon, S. Lem, S. R. Millon, J. Q. Brown, V. L. Seewaldt, N. Ramanujam, Cancer Res. 2010, 70(11), 4759.

[31] K. Staniszewski, S. H. Audi, R. Sepehr, E. R. Jacobs, M. Ranji, Ann. Biomed. Eng. 2013, 41(4), 827.

[32] V. Shupletsov, K. Kandurova, E. Seryogina, G. Piavchenko, V. Dremin, A. Mamoshin, A. Dunaev, Proc. SPIE 2019, 11140, 1114001-212.

[33] V. Dremin, E. Potapova, A. Mamoshin, A. Dunaev, E. Rafailov, Laser Phys. Lett 2020, 17(11), 115605.

[34] B. Wilson, S. Jacques, IEEE J Quant Electron 1990, 26(12), 2186.

[35] A. I. Zherebtsova, V. Dremin, I. N. Makovik, E. A. Zherebtsov, A. V. Dunaev, A. Goltsov, S. G. Sokolovski, E. U. Rafailov, Front. Physiol. 2019, 10, 416.

[36] V. V. Dremin, E. A. Zherebtsov, V. V. Sidorov, A. Krupatkin, I. N. Makovik, A. I. Zherebtsova, E. V. Zharkikh, E. V. Potapova, A. V. Dunaev, A. A. Doronin, A. V. Bykov, I. E. Rafailov, K. S. Litvinova, S. G. Sokolovski, E. U. Rafailov, J Biomed Opt. 2017, 22(8), 1.

How to cite this article: G. Piavchenko, I. Kozlov, V. Dremin, D. Stavtsev, E. Seryogina, K. Kandurova, V. Shupletsov, K. Lapin, A. Alekseyev, S. Kuznetsov, A. Bykov, A. Dunaev, I. Meglinski, J. Biophotonics 2021, 14(12), e202100216. https://doi.org/10.1002/jbio.202100216 